D.T. Wickramasinghe, L. Ferrario, and G.V. Bicknell, eds.

\title{
Eclipse Mapping of the Accretion Disk Wind in the Cataclysmic Variable UX UMa
}

\author{
Christian Knigge ${ }^{1}$, Janet E. Drew ${ }^{2}$, Keith O. Mason ${ }^{3}$ and Knox S. Long ${ }^{1}$ \\ ${ }^{1}$ Space Telescope Sciences Institute, 3700 San Martin Drive, Baltimore \\ MD 21218, USA \\ ${ }^{2}$ Imperial College, Blackett Laboratory, Prince Consort Road, London \\ $S W 7$ 2BZ, UK \\ ${ }^{3}$ Mullard Space Science Laboratory, University College London, \\ Holmbury St. Mary, Dorking, Surrey RH5 6NT, UK
}

\begin{abstract}
We present the results of an effort to model recent HST eclipse observations of the wind-formed C IV $1550 \AA$ resonance line in the high-inclination nova-like variable UX UMa (Mason et al. 1995, Baptista et al. 1995). Within the framework of a simple kinematic model, in which the outflow is described as a rotating, biconical accretion disk wind (Knigge et al. 1995), we are able to reproduce not only the shapes and strengths of the observed line profiles both away from and during eclipse, but also most of the detailed behaviour of different parts of the line (blue wing, line centre and red wing) as a function of orbital phase (Figure 1).

The most important result of our modeling is that it strongly suggests the presence of a vertically extended $\left(H \sim f e w \times 10 R_{W D}\right)$, relatively dense $\left(n_{e} \sim 10^{13} \mathrm{~cm}^{-3}\right)$ and only slowly-outflowing ( $v_{\text {poloidal }}<<v_{\text {escape }}$ ) transition region between the disk photosphere and the fast-moving wind. Thus the best current data already demand that the simplified picture of accretion disks and winds as quasi-independent physical phenomena should be replaced by a unified theory of accretion disk atmospheres. It may be important in this context that, barring strong contamination of the observed $\mathrm{C}$ IV wind line by an underlying disk-formed component, the evidence for rotation in the outflow is unambiguous: the observed line flux light curves clearly exhibit a rotational disturbance that is well matched by our simple model of a rotating disk wind.
\end{abstract}

\section{References}

Baptista, R., Horne, K., Hilditch, R.W., Mason, K.O. \& Drew, J.E. 1995, ApJ, 395,448

Knigge, C., Woods, J.A. \& Drew, J.E., 1995, MNRAS, 273, 225

Mason, K.O., Drew, J.E., Córdova, F.A., Horne, K., Hilditch, R., Knigge, C., Lanz, T. \& Meylan, T. 1995, MNRAS, 274, 271 

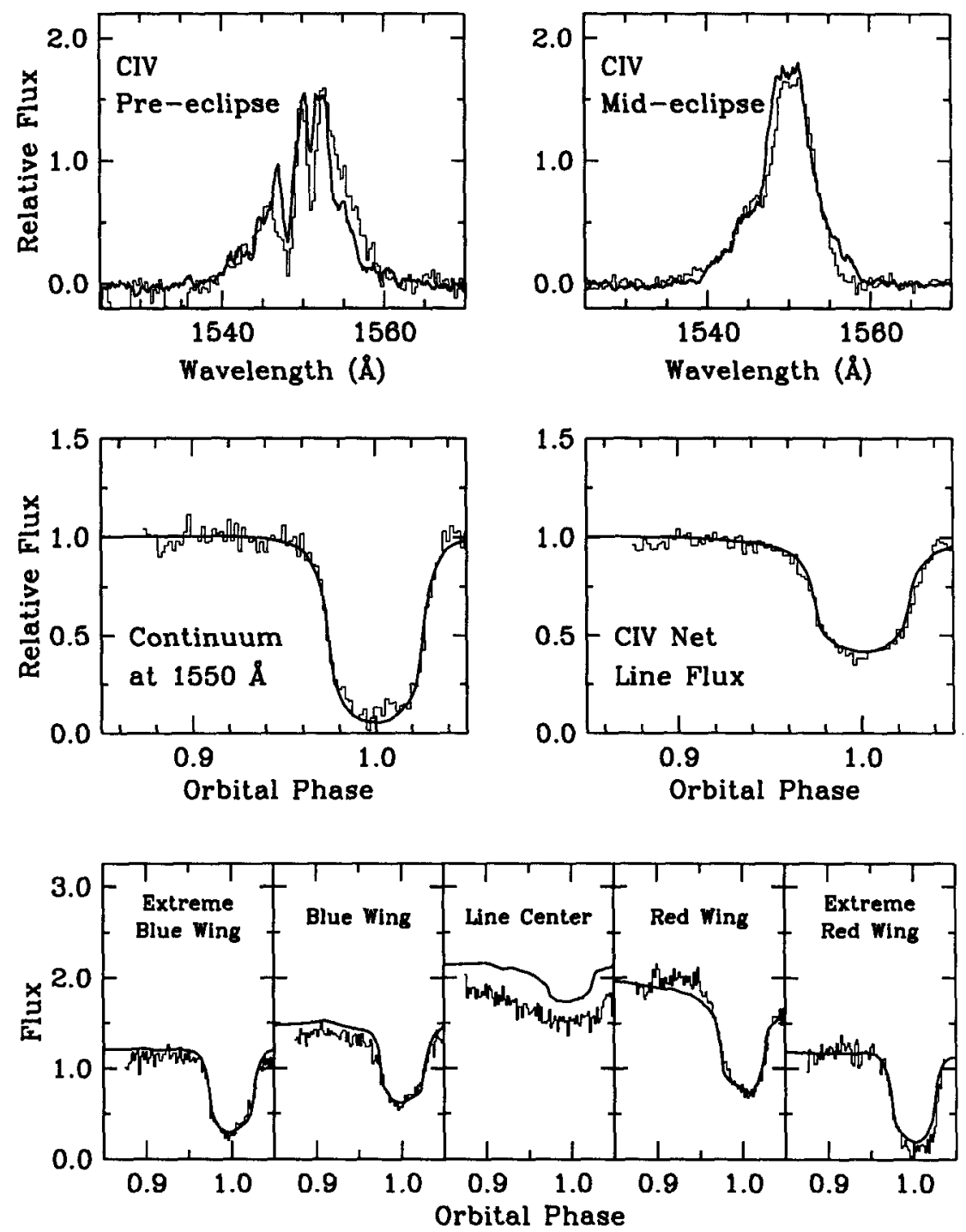

Figure 1. The eclipse behaviour of the best-bet disk wind model (thick lines) compared to the observations (thin histograms).

Top Panels: Normalized and continuum subtracted pre- (left panel) and mid-eclipse (right panel) C IV line profiles.

Middle Panels: Normalized continuum (left panel) and net $\mathrm{C}$ IV line flux (right panel) light curves.

Bottom Panels: Light curves of isolated parts of the C IV line. 